Bangladesh J. Pl. Breed. Genet., 28(1): 09-16, 2015

\title{
ESTIMATION OF STANDARD HETEROSIS FOR REPRODUCTIVE TRAITS IN NEWLY SYNTHESIZED TEST RICE (Oryza sativa L.) HYBRIDS
}

\author{
M. Ariful Islam ${ }^{2 *}$, M. A. K. Mian ${ }^{1}$, M. G. Rasul ${ }^{1}$ and M. K. Bashar ${ }^{3}$ \\ ${ }^{1}$ Department of Genetics and Plant Breeding, Bangabandhu Sheikh Mujibur Rahman \\ Agricultural University, Gazipur 1706, Bangladesh \\ ${ }^{2}$ Department of Genetics and Plant Breeding, Exim Bank Agricultural University, \\ Chapainawabgonj, Bangladesh \\ ${ }^{3}$ GRS Division, Bangladesh Rice Research Institute, Gazipur 1701, Bangladesh
}

\begin{abstract}
An experiment was carried to estimate heterotic performances of newly developed test hybrids utilizing CMS system during Rabi 2011 using RCBD design. Five known CMS lines and sixteen developed R-lines were crossed through line $\times$ tester analysis. Heterosis for days to first flowering varied from $6.23 \%$ to $16.35 \%$ with a SD of 4.47 meanwhile heterosis for days to $80 \%$ flowering varied from $-9.24 \%$ to $11.04 \%$ with a SD of 4.03 . Among eighty crosses seventy two were found positive heterosis for days to first flowering but sixty four crosses were found for days to $80 \%$ flowering. RG-BU08-016R crosses with IR58025A $(-2.68 *)$ and IR62820A $(-3.42 * *)$ showed significant negative standard heterosis for days to first flowering. Five crosses of IR58025A showed significant negative heterosis with RG-BU 08-005R (3.42*), RG-BU 08-006R (-5.28**), RG-BU 08-013R (-4.129**), RG-BU 08016R (-5.78**), RG-BU 08-018R (-4.06*) for days to $80 \%$ flowering. Besides these 4 crosses of IR62820A showed significant negative heterosis with RGBU 08-007R (-2.32*), RG-BU 08-016R (-6.03*), RG-BU 08-018R (-4.58**) followed by GAN 46A/RG-BU 08-006R (-4.58*), BRRI 1A/RG-BU 08-001R $\left(-2.55^{* *}\right)$ and BRRI 1A/RG-BU 08-006R (-9.24**) resulted significant negative heterosis for days to $80 \%$ flowering. Positive heterosis were recorded for IR58025A with RG-BU 08-007R (26.97**), RG-BU 08-0046R (8.77**) and RG-BU 08-105R (14.44**) for PER and RG-BU 08-018R (42.46**), RG-BU 08-046R (112.30**), RG-BU 08-057R (27.46*) for SER. While two R-lines, RG-BU 08-013R (25.41**) and RG-BU 08-057R (7.21*) were found having significant positive heterosis for panicle exertion rate crosses with IR62820A but RG-BU 08-025R (52.82**), RG-BU 08-057R $\left(122.66^{* *}\right)$ and RG-BU 08-063R (37.38**) were found having significant positive heterosis for SER. IR62820A/RG-BU08-057R showed significant positive heterosis for both panicle and stigma exertion rate. GAN46A/RGBU 08-063R $\left(5.65^{*} \& 133.02 * *\right)$ was recorded showing significant positive heterosis for both panicle and stigma exertion rate where. But the crosses of IR68888A was not shown exhibiting a great contribution for panicle and stigma exertion rate except RG-BU08-018R (25.94**). Meanwhile the crosses of BRRI1A was recorded having significant positive heterosis for PER with RG-BU08-005R (13.41**), RG-BU08-007R (5.65*) and RGBU08-097R (25.94**) as well as RG-BU08-007R (133.02**), RG-BU08013R (48.19**) and RG-BU08-038R (29.21*) were recorded having significant positive heterosis for SER.
\end{abstract}

Keywords: Rice; heterosis; days to flowering; panicle exertion; stigma exertion

DOI: http://dx.doi.org/10.3329/bjpbg.v28i1.27841 


\section{INTRODUCTION}

Heterosis is an important genetic phenomenon, synonymous with hybrid vigour refers to the manifested superiority of the $F_{1}$ hybrid resulting from the cross of genetically dissimilar homozygous parents. The best way to utilize heterosis in crop is to produce $\mathrm{F}_{1}$ hybrids, which possess maximum heterozygosity (Chetia et al., 2000). Heterosis in rice has been known since 1926, its commercial exploitation was demonstrated only when Chinese rice scientists developed commercial rice hybrids with 20 percent yield advancement over semi-dwarf varieties (Sun et al., 2006). Heterosis is estimated to determine the superiority of $\mathrm{F}_{1}$ over mid parent or better parent or standard check variety (Hien et al., 2007). Moreover yield potentialities of HYV are in static position.

Therefore, there is an ample scope of enhancement of yield potentiality of rice through development of hybrid variety utilizing CMS system in the country. Nonetheless, the geographical location of Bangladesh is quite favorable for hybrid rice production (Banumathy and Thiyagarajan, 2005). Evaluation of heterosis is a crucial part of hybrid breeding. Heterosis will help the breeder to observe superiority of the hybrids in respect of their parents or standard rice varieties. The observation related to heterosis of different characters of rice showed both positive and negative values (Chahal and Gosal, 2008).

Considering above ideas the present investigation was undertaken with the following objectives: (i) to develop short duration variety; (ii) to estimate standard heterosis for panicle and stigma exertion rate and (iii) to estimate standard heterosis for yield in newly synthesized test hybrids.

\section{MATERIALS AND METHODS}

The experiment was carried out at the experimental farm, Department of Genetics and Plant Breeding, BSMRAU, Gazipur 1706 during Rabi 2011. The experimental site is located at the centre of Madhupur Tract $\left(24^{\circ} 29 \mathrm{~N}\right.$ latitude and $90^{\circ} 26 \mathrm{E}$ longitude) having an elevation of $8.2 \mathrm{~m}$ from the sea level. The soil type of experimental field belongs to the shallow red brown terrace type under Salna series of Madhupur Tract (AEZ- 28) which is characterized by sandy clay with $\mathrm{p}^{\mathrm{H}}$ value of 6.5. 5 known CMS lines and 16 developed R-lines were crossed through line $\times$ tester analysis and 80 test hybrids were developed. These 80 test hybrids were sown in the subsequent generation to assess heterotic performances in randomized complete block design.

Data on the following parameters were recorded from 10 randomly selected plants and on whole plot basis on days to $1^{\text {st }}$ flowering, days to $80 \%$ flowering, panicle exertion rate (\%PER), stigma exertion rate (\%PER) and finally grain yield.

Data obtained for each character was subjected to the analysis of variance following three replicated randomized complete block design by using MSTAT-C computer software and the analysis of variance for each of the characters was performed by F-test. Mean, range and standard deviation for each character were also estimated for the hybrids performed by Microsoft Excel program 2007. Standard heterosis was calculated as percentage increase or decrease of $F_{1}$ mean performance over the check variety. 


\section{Materials used in the experiment}

Table 1. Male Parents, Female, Parents and the $F_{1} s$ used for standard heterosis estimation over BRRI dhan 28

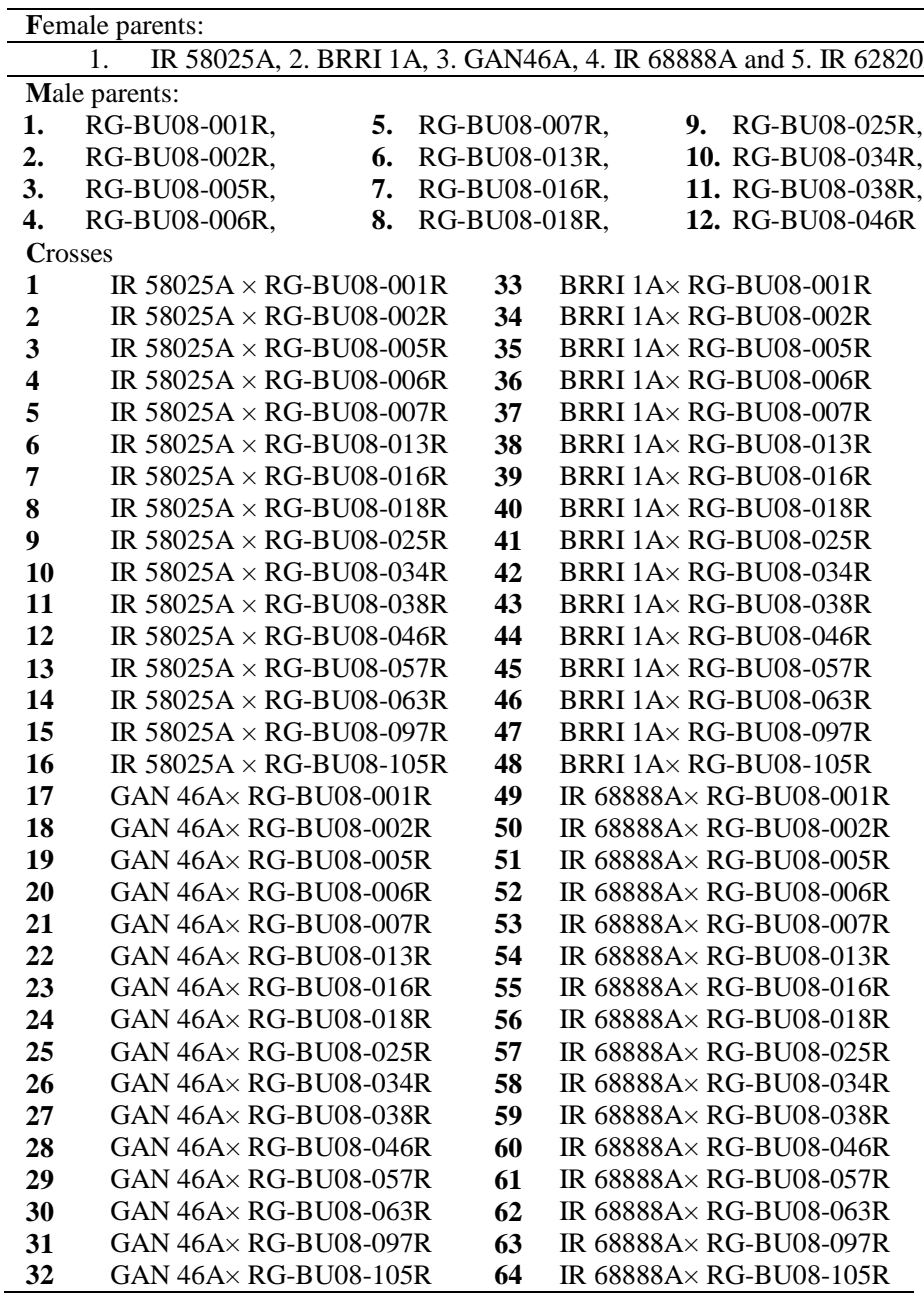

\section{Estimation of Standard Heterosis}

Mean value of check variety and $F_{1} S$ in all replications were taken for heterosis estimation.

$$
\begin{array}{cl}
\text { Hs \% }=\left(\mathrm{F}_{1}-\mathrm{CV}\right) / \mathrm{CV} \times 100 & \text { Where } \\
\mathrm{SE}(\mathrm{Hs})=\frac{(2 \mathrm{Ve})}{\mathrm{r}} & \text { Ve }=\text { Error mean sum of squares from RCBD } \\
\text { "t" value }=\mathrm{F}_{1}-\mathrm{CV} / \mathrm{SE}(\mathrm{Hs}) & \text { ANOVA, } \\
& \mathrm{F}_{1}=\text { Mean of } \mathrm{F}_{1}, \\
& \mathrm{CV}=\text { Mean of check variety, } \\
& \mathrm{Hs}=\text { Standard heterosis, } \\
& \mathrm{SE}=\text { Standard error }
\end{array}
$$

65 IR 62820 A $\times$ RG-BU08-001R

66 IR 62820 A $\times$ RG-BU08-002R

67 IR 62820 A $\times$ RG-BU08-005R

IR 62820 A $\times$ RG-BU08-006R

70 IR 62820 A $\times$ RG-BU08-013R

71 IR $62820 \mathrm{~A} \times$ RG-BU08-016R

72 IR 62820A $\times$ RG-BU08-018R

IR $62820 \mathrm{~A} \times$ RG-BU08-025R

62820A× RG-BU08-034R

76 IR $62820 \mathrm{~A} \times \mathrm{RG}$ R-BU08-038R

77 IR 62820A $\times$ RG-BU08-057R

78 IR $62820 A \times$ RG-BU08-063R

IR 62820A× RG-BU08-097R

80 IR $62820 A \times$ RG-BU08-105R 


\section{RESULTS AND DISCUSSION}

\section{Estimation of standard heterosis for days to first and $80 \%$ flowering}

BRRI dhan28 is an outstanding variety due to its fine grain shape and earliness in nature. It is very difficult to find a hybrid earlier to BRRI dhan28 as life cycle of 140 days. Heterosis for days to first flowering varied from $-6.23 \%$ to $16.35 \%$ with a SD of 4.47 meanwhile heterosis for days to $80 \%$ flowering varied from $-9.24 \%$ to $11.04 \%$ with a SD of 4.03. Among eighty crosses seventy two crosses were found positive heterosis for days to first flowering but 64 were found for days to $80 \%$ flowering. Normally the number of crosses for first flowering and $80 \%$ flowering are same but deviation might be due to prolongs flowering period. Some crosses completed their flowering period within 7 days but some crosses took 12-17 days for completion of flowering. This might be due to abiotic factor (effect of photoperiod, temperature and humidity) and inaccuracy of pollination.

Table 02. Estimation of standard heterosis over BRRI dhan28 for days to first flowering in newly developed $80 \mathrm{~F}_{1}$ hybrids

\begin{tabular}{|c|c|c|c|c|c|}
\hline \multirow{2}{*}{ Crosses } & \multicolumn{5}{|c|}{ Days to first flowering } \\
\hline & IR 58025A & IR 62829A & GAN 46 A & IR 68888A & BRRI 1A \\
\hline RG-BU 08-001R & $5.88 \pm 3.32$ & $25.74 * * \pm 3.75$ & $25.74 * * \pm 3.60$ & $15.81^{* *} \pm 3.22$ & $2.82^{* *} \pm 4.01$ \\
\hline RG-BU 08-002R & $11.99 * * \pm 3.32$ & $16.33^{* *} \pm 3.75$ & $16.33 * * \pm 3.60$ & $13.53 * * \pm 3.22$ & $31.88 * * \pm 4.01$ \\
\hline RG-BU 08-005R & $0.52 \pm 3.32$ & $13.53^{* *} \pm 3.75$ & $13.53 * * \pm 3.60$ & $11.23 * * \pm 3.22$ & $36.45^{* *} \pm 4.01$ \\
\hline RG-BU 08-006R & $-4.29 \pm 3.32$ & $-16.85^{* *} \pm 3.75$ & $-2.54 \pm 3.60$ & $8.93 * * \pm 3.22$ & $-14.77 * * \pm 4.01$ \\
\hline RG-BU 08-007R & $20.90 * * \pm 3.32$ & $2.06 \pm 3.75$ & $18.11 * * \pm 3.60$ & $11.23 * * \pm 3.22$ & $22.70 * * \pm 4.01$ \\
\hline RG-BU 08-013R & $-1.78 \pm 3.32$ & $8.06^{*} \pm 3.75$ & $10.48 * * \pm 3.60$ & $22.70 * * \pm 3.22$ & $22.70^{* *} \pm 4.01$ \\
\hline RG-BU 08-016R & $-6.35^{*} \pm 3.32$ & $-8.11 \pm 3.75$ & $15.81 * * \pm 3.60$ & $20.41 * * \pm 3.22$ & $29.58 * * \pm 4.01$ \\
\hline RG-BU 08-018R & $-2.54 \pm 3.32$ & $-2.54 \pm 3.75$ & $13.46 * * \pm 3.60$ & $11.23 * * \pm 3.22$ & $34.18 * * \pm 4.01$ \\
\hline RG-BU 08-025R & $2.82 \pm 3.32$ & $2.65 \pm 3.75$ & $38.75 * * \pm 3.60$ & $15.81 * * \pm 3.22$ & $21.92 * * \pm 4.01$ \\
\hline RG-BU 08-034R & $-8.18 * \pm 3.32$ & $8.39^{*} \pm 3.75$ & $22.70 * * \pm 3.60$ & $18.11^{* *} \pm 3.22$ & $-12.75^{* *} \pm 4.01$ \\
\hline RG-BU 08-038R & $14.03 * * \pm 3.32$ & $13.79 * * \pm 3.75$ & $22.70 * * \pm 3.60$ & $20.41 * * \pm 3.22$ & $9.69 * * \pm 4.01$ \\
\hline RG-BU 08-046R & $15.05^{* *} \pm 3.32$ & $14.95 * * \pm 3.75$ & $29.58 * * \pm 3.60$ & $15.81^{* *} \pm 3.22$ & $15.81 * * \pm 4.01$ \\
\hline RG-BU 08-057R & $22.70 * * \pm 3.32$ & $22.59 * * \pm 3.75$ & $34.18 * * \pm 3.60$ & $13.53 * * \pm 3.22$ & $13.56^{* *} \pm 4.01$ \\
\hline RG-BU 08-063R & $21.16^{* *} \pm 3.32$ & $21.26^{* *} \pm 3.75$ & $21.92 * * \pm 3.60$ & $27.28 * * \pm 3.22$ & $13.51 * * \pm 4.01$ \\
\hline RG-BU 08-097R & $7.28 * * \pm 3.32$ & $-7.26 * * \pm 3.75$ & $2.75^{* *} \pm 3.60$ & $2.70 * * \pm 3.22$ & $-7.28 * * \pm 4.01$ \\
\hline RG-BU 08-105R & $18.87 * * \pm 3.32$ & $16.73 * * \pm 3.75$ & $-9.69 * * \pm 3.60$ & $20.41 * * \pm 3.22$ & $22.70^{* *} \pm 4.01$ \\
\hline Minimum & 38.75 & & & & \\
\hline Maximum & -14.77 & & & & \\
\hline SD & 10.628 & & & & \\
\hline SE & 3.329 & & & & \\
\hline $\mathbf{t}(5 \%) / \mathrm{SE}(\mathrm{gi})$ & 6.036 & & & & \\
\hline $\mathbf{t}(1 \%) / \mathrm{SE}(\mathrm{gi}-\mathrm{gj})$ & 8.714 & & & & \\
\hline
\end{tabular}

It is to be noted that only eleven crosses were found exhibiting negative heterosis among which only two crosses were found significantly earlier than BRRI dhan 28 in context of days to first flowering. RG-BU08-016R crosses with IR58025A $\left(-2.68^{*}\right)$ and IR62820A $(-3.42 * *)$ showed significant negative standard heterosis for days to first flowering. 5 crosses of IR58025A showed significant negative heterosis with RG-BU 08-005R (-3.42*), RG-BU 08-006R (-5.28**), RG-BU 08-013R (-4.129**), RG-BU 08-016R (-5.78**), RG-BU 08-018R (-4.06*) for days to $80 \%$ flowering. Besides these 4 crosses of IR62820A showed significant negative heterosis with RG-BU 08-007 R (2.32*), RG-BU 08-016R (-6.03*), RG-BU 08-018R (-4.58**) followed by GAN 46A/RG-BU 08-006R (-4.58*), BRRI 1A/RG-BU 08-001R $\left(-2.55^{* *}\right)$ and BRRI 1A/RG-BU 08-006R (-9.24**) resulted significant negative heterosis for days to $80 \%$ flowering. 
Biju et al. (2006) observed magnitude of standard heterosis and per se performance of thirty four hybrids and found significant negative heterosis for plant height and days to $50 \%$ flowering. Bhave et al. (2002) evaluated thirty-two rice hybrids from two CMS lines for heterosis (mid-parent) and standard heterosis and observed 58.50 to $80.43 \%$ heterosis for days to maturity. Ali (2007) while studying on fifty test hybrid demonstrated that hybrid-4 and hybrid- 9 exhibited all types of heterosis in desirable direction for most of the characters, especially for days to $80 \%$ flowering and days to maturity. Roy (2006) found ten specific cross combinations performed excellent and most promising hybrids showed positive significant negative heterosis for days to $80 \%$ flowering and maturity. In fact from this table it might be concluded that there is few possibilities to found test hybrid having a significant negative heterosis compared to BRRI dhan28.

Table 03. Estimates of standard heterosis over BRRI dhan 28 for days to days to $80 \%$ flowering in newly developed $80 \mathrm{~F}_{1}$ hybrids

\begin{tabular}{|c|c|c|c|c|c|}
\hline \multirow{2}{*}{ Crosses } & \multicolumn{5}{|c|}{ Days to $80 \%$ flowering } \\
\hline & IR 58025A & IR 62829A & GAN 46 A & IR 68888A & BRRI 1A \\
\hline RG-BU 08-001R & $7.61 * \pm 3.30$ & $26.81 * * \pm 4.55$ & $24.01 * * \pm 4.07$ & $15.08 * * \pm 3.22$ & $1.09 \pm 3.77$ \\
\hline RG-BU 08-002R & $13.72 * * \pm 3.30$ & $17.40^{* *} \pm 4.55$ & $14.60 * * \pm 4.07$ & $12.80 * * \pm 3.22$ & $30.15 * * \pm 3.77$ \\
\hline RG-BU 08-005R & $2.25 \pm 0.30$ & $14.60 * * \pm 4.55$ & $11.80 * * \pm 4.07$ & $10.50 * * \pm 3.22$ & $34.72 * * \pm 3.77$ \\
\hline RG-BU 08-006R & $-2.56 \pm 0.30$ & $-17.92 * * \pm 4.55$ & $-4.27 \pm 4.07$ & $8.20 * \pm 3.22$ & $-16.50 * * \pm 3.77$ \\
\hline RG-BU 08-007R & $22.63 * * \pm 3.30$ & $3.13 \pm 0.55$ & $16.38 * * \pm 4.07$ & $10.50 * * \pm 3.22$ & $20.97 * * \pm 3.77$ \\
\hline RG-BU 08-013R & $-0.95 \pm 0.30$ & $9.13 * * \pm 4.55$ & $8.75^{* *} \pm 4.07$ & $21.97 * * \pm 3.22$ & $20.97 * * \pm 3.77$ \\
\hline RG-BU 08-016R & $-4.62 \pm 0.30$ & $-7.04 * \pm 4.55$ & $14.08 * * \pm 4.07$ & $19.68 * * \pm 3.22$ & $27.85^{* *} \pm 3.77$ \\
\hline RG-BU 08-018R & $-0.81 \pm 0.30$ & $-1.47 \pm 0.55$ & $11.73 * * \pm 4.07$ & $10.50 * * \pm 3.22$ & $32.45^{* *} \pm 3.77$ \\
\hline RG-BU 08-025R & $4.55 \pm 3.30$ & $3.72 \pm 0.55$ & $37.02 * * \pm 4.07$ & $15.08 * * \pm 3.22$ & $20.19 * * \pm 3.77$ \\
\hline RG-BU 08-034R & $-9.91 * \pm 3.30$ & $9.25 * * \pm 4.55$ & $20.97 * * \pm 4.07$ & $17.38 * * \pm 3.22$ & $-11.02 * * \pm 3.77$ \\
\hline RG-BU 08-038R & $15.76^{* *} \pm 3.30$ & $14.86^{* *} \pm 4.55$ & $20.97 * * \pm 4.07$ & $19.68 * * \pm 3.22$ & $7.96 * \pm 3.77$ \\
\hline RG-BU 08-046R & $16.78 * * \pm 3.30$ & $16.02 * * \pm 4.55$ & $27.85 * * \pm 4.07$ & $15.08 * * \pm 3.22$ & $14.08 * * \pm 3.77$ \\
\hline RG-BU 08-057R & $24.43 * * \pm 3.30$ & $23.66^{* *} \pm 4.55$ & $32.45 * * \pm 4.07$ & $12.80 * * \pm 3.22$ & $11.83 * * \pm 3.77$ \\
\hline RG-BU 08-063R & $22.89 * * \pm 3.30$ & $22.33 * * \pm 4.55$ & $20.19 * * \pm 4.07$ & $26.55^{* *} \pm 3.22$ & $11.78 * * \pm 3.77$ \\
\hline RG-BU 08-097R & $9.01 * * \pm 3.30$ & $-8.33 * * \pm 4.55$ & $-9.02 * * \pm 4.07$ & $1.97 * * \pm 3.22$ & $-5.55 * * \pm 3.77$ \\
\hline RG-BU 08-105R & $20.60 * * \pm 3.30$ & $17.80 * * \pm 4.55$ & $-7.96^{*} \pm 4.07$ & $19.68 * * \pm 3.22$ & $20.97 * * \pm 3.77$ \\
\hline Minimum & $\mathbf{3 7 . 0 2}$ & & & & \\
\hline Maximum & -16.50 & & & & \\
\hline SD & 10.197 & & & & \\
\hline SE & $\mathbf{3 . 5 7 2}$ & & & & \\
\hline t (5\%)/SE (gi) & 6.833 & & & & \\
\hline $\mathbf{t}(1 \%) / \mathrm{SE}(\mathrm{gi}-\mathrm{gj})$ & 8.444 & & & & \\
\hline
\end{tabular}

\section{Estimation of standard heterosis for panicle exertion rate (\%PER) and stigma exertion rate (\%SER)}

Highly significant positive heterosis for panicle and stigma exertion rate indicates greater suitability of the cross combination over check varieties. Among 80 crosses, positive heterosis were recorded for the crosses of IR58025A with RG-BU 08-007R (26.97**), RG-BU 08-0046R (8.77**) and RG-BU 08-105R (14.44**) for panicle exertion rate and RG-BU 08-018R (42.46**), RG-BU 08-046R (112.30**), RG-BU 08-057R (27.46*) for stigma exertion rate. While two R-lines, RG-BU 08013R $\left(25.41^{* *}\right)$ and RG-BU 08-057R (7.21*) were found having significant positive heterosis for panicle exertion rate crosses with IR62820A but RG-BU 08-025R (52.82**), RG-BU 08-057R (122.66**) and RG-BU 08-063R (37.38**) were found having significant positive heterosis for stigma exertion rate. Here it is to be noted that IR62820A/RG-BU08-057R showed significant positive heterosis for both 
panicle and stigma exertion rate. GAN46A/RG-BU08-063R (5.65* \& 133.02**) was recorded showing significant positive heterosis for both panicle and stigma exertion rate where. But the crosses of IR68888A was not shown exhibiting a great contribution for panicle and stigma exertion rate except RG-BU08-018R (25.94**).

Table 5. Estimation of standard heterosis over BRRI dhan28 for panicle exertion rate (\% PER) in $80 \mathrm{~F}_{1}$

\begin{tabular}{|c|c|c|c|c|c|}
\hline \multirow[t]{2}{*}{ Crosses } & \multicolumn{5}{|c|}{ Panicle exertion rate (\% PER) } \\
\hline & IR 58025A & IR 62820A & GAN 46A & IR $68888 \mathrm{~A}$ & BRRI 1A \\
\hline RG-BU 08-001R & $2.07 \pm 0.95$ & $1.00 \pm 0.83$ & $12.87 * * \pm 0.85$ & $-5.79 * \pm 0.78$ & $1.02 \pm 0.80$ \\
\hline RG-BU 08-002R & $3.85 \pm 0.95$ & $0.51 \pm 0.83$ & $-0.56 \pm 0.85$ & $-10.02 * * \pm 0.78$ & $-3.69 \pm 0.80$ \\
\hline RG-BU 08-005R & $-4.63 \pm 0.95$ & $2.29 \pm 0.83$ & $-1.05 \pm 0.85$ & $-2.12 \pm 0.78$ & $13.41 * * \pm 0.80$ \\
\hline RG-BU 08-006R & $-3.83 \pm 0.95$ & $-6.20 * \pm 0.83$ & $0.72 \pm 0.85$ & $1.04 \pm 0.78$ & $-0.03 \pm 0.80$ \\
\hline RG-BU 08-007R & $26.97 * * \pm 0.95$ & $-5.40 * \pm 0.83$ & $-7.76 * \pm 0.85$ & $2.82 \pm 0.78$ & $5.65^{*} \pm 0.80$ \\
\hline RG-BU 08-013R & $-2.17 \pm 0.95$ & $25.41 * * \pm 0.83$ & $-6.96 * \pm 0.85$ & $-5.66^{*} \pm 0.78$ & $-12.11 * * \pm 0.80$ \\
\hline RG-BU 08-016R & $-9.00 * * \pm 0.95$ & $-3.73 \pm 0.83$ & $23.84 * * \pm 0.85$ & $-4.86^{*} \pm 0.78$ & $-1.08 \pm 0.80$ \\
\hline RG-BU 08-018R & $-2.97 \pm 0.95$ & $-10.56^{* *} \pm 0.83$ & $-5.29 * \pm 0.85$ & $25.94 * * \pm 0.78$ & $-5.79 * \pm 0.80$ \\
\hline RG-BU 08-025R & $-3.42 \pm 0.95$ & $-4.53^{*} \pm 0.83$ & $-12.13 * * \pm 0.85$ & $-3.20 \pm 0.78$ & $11.31 \pm 0.80$ \\
\hline RG-BU 08-034R & $-4.89 * \pm 0.95$ & $-4.98^{*} \pm 0.83$ & $-6.09 * \pm 0.85$ & $-10.03 * * \pm 0.78$ & $-2.12 \pm 0.80$ \\
\hline RG-BU 08-038R & $1.41 \pm 0.95$ & $-6.45^{*} \pm 0.83$ & $-6.54 * \pm 0.85$ & $-4.00 \pm 0.78$ & $1.04 \pm 0.80$ \\
\hline RG-BU 08-046R & $8.77 * * \pm 0.95$ & $-0.15 \pm 0.83$ & $-8.01 * * \pm 0.85$ & $-4.44 \pm 0.78$ & $2.82 \pm 0.80$ \\
\hline RG-BU 08-057R & $-8.99 * * \pm 0.95$ & $-4.23 \pm 0.83$ & $-1.71 \pm 0.85$ & $-5.92 * \pm 0.78$ & $-5.66^{*} \pm 0.80$ \\
\hline RG-BU 08-063R & $2.04 \pm 0.95$ & $-10.55 * * \pm 0.83$ & $-1.08 \pm 0.85$ & $0.38 \pm 0.78$ & $-4.86^{*} \pm 0.80$ \\
\hline RG-BU 08-097R & $14.44 * * \pm 0.95$ & $7.21^{*} \pm 0.83$ & $5.65 * \pm 0.85$ & $11.31 * * \pm 0.78$ & $25.94 * * \pm 0.80$ \\
\hline RG-BU 08-105R & $-2.67 \pm 0.95$ & $0.48 \pm 0.83$ & $-12.11 * * \pm 0.85$ & $7.75 \pm 0.78$ & $-3.20 \pm 0.80$ \\
\hline Minimum & $-12.13 * *$ & & & & \\
\hline Maximum & $26.97 * *$ & & & & \\
\hline SD & 8.74 & & & & \\
\hline t $(5 \%) / \mathrm{SE}(\mathrm{gi})$ & 0.97 & & & & \\
\hline $\mathbf{t}(1 \%) / \mathrm{SE}(\mathrm{gi}-\mathrm{gj})$ & 4.459 & & & & \\
\hline
\end{tabular}

${ }^{*} \mathrm{p}=0.05, * * \mathrm{p}=0.01$ and $^{\mathrm{ns}}=$ Insignificant

Meanwhile the crosses of BRRI1A was recorded having significant positive heterosis for panicle exertion rate with RG-BU08-005R (13.41**), RG-BU08-007R (5.65*) and RG-BU08-097R (25.94**) as well as RG-BU08-007R (133.02**), RGBU08-013R (48.19**) and RG-BU08-038R (29.21*) recorded having significant positive heterosis for stigma exertion rate which reveals that among all the cross combinations these crosses exceeded the standard variety. The higher panicle and stigma exertion might be due to synchronized flowering due to application of growth inhibiting substances within virgin panicle initiation stage (PI-6) to heading. The higher panicle exertion might also be due to proper application of micronutrients $\left(\mathrm{GA}_{3}\right)$ just before panicle initiation stage as well as active tillering stage @ $12 \mu \mathrm{m}$ as dose $01,24 \mu \mathrm{m}$ as dose $02,36 \mu \mathrm{m}$ as dose 03 and $48 / 54 \mu \mathrm{m}$ as dose 04 . It is to be noted that dose $04(48 / 54 \mu \mathrm{m})$ is used depending on environment and reproductive phase of the panicle initiation stage. Banumathy et al. (2005) studied the magnitude of heterosis, of 100 rice hybrids for grain yield and its related traits and observed maximum significant positive relative heterosis (205.29\%) and heterobeltiosis (187.25\%). He also stated that most of the high yielding hybrids manifested positive heterosis for filled grains and spikelet fertility. Meanwhile, Bhave et al. (2002) evaluated thirty-two rice hybrids from two CMS lines (IR58025A and IR62829A) and 16 local restorers and found that standard heterosis -58.50 to $80.43 \%$, respectively for spikelets fertility. 
Table 6. Estimation of standard heterosis over BRRI dhan28 for stigma exertion rate (\% SER) in $80 \mathrm{~F}_{1}$

\begin{tabular}{|c|c|c|c|c|c|}
\hline \multirow[t]{2}{*}{ Crosses } & \multicolumn{5}{|c|}{ Stigma exertion rate (\% SER) } \\
\hline & IR 58025A & IR $62820 \mathrm{~A}$ & GAN 46A & IR $68888 \mathrm{~A}$ & BRRI 1A \\
\hline RG-BU 08-001R & $-68.83^{* *} \pm 0.91$ & $-8.68 \pm 0.87$ & $-5.39 \pm 2.85$ & $-69.17 * * 2.22$ & $7.22 \pm 1.05$ \\
\hline RG-BU 08-002R & $-14.74 \pm 0.91$ & $-58.47 * * \pm 0.87$ & $1.68 \pm 2.85$ & $-79.28 * * \pm 2.22$ & $8.15 \pm 1.05$ \\
\hline RG-BU 08-005R & $-74.39 * * \pm 0.91$ & $-4.38 \pm 0.87$ & $-48.10^{* *} \pm 2.85$ & $-72.20 * * \pm 2.22$ & $-1.96 \pm 1.05$ \\
\hline RG-BU 08-006R & $-15.75 \pm 0.91$ & $-64.03 * * \pm 0.87$ & $5.98 \pm 2.85$ & $-55.04 * * \pm 2.22$ & $-79.13 * * \pm 1.05$ \\
\hline RG-BU 08-007R & $-80.03 * \pm 0.91$ & $-5.39 \pm 0.87$ & $-53.66^{* *} \pm 2.85$ & $-0.95 \pm 2.22$ & $-66.24 * * \pm 1.05$ \\
\hline RG-BU 08-013R & $-48.10 * * \pm 0.91$ & $-69.67 * * \pm 0.87$ & $4.97 \pm 2.85$ & $-60.60 * * \pm 2.22$ & $48.19^{* *} \pm 1.05$ \\
\hline RG-BU 08-016R & $2.44 \pm 0.91$ & $-37.74 * * \pm 0.87$ & $-59.31 * * \pm 2.85$ & $-1.96 \pm 2.22$ & $14.15 \pm 1.05$ \\
\hline RG-BU 08-018R & $27.46^{*} \pm 0.91$ & $37.83^{* *} \pm 0.87$ & $48.19^{* *} \pm 2.85$ & $126.09 * * \pm 2.22$ & $133.02 * * \pm 1.05$ \\
\hline RG-BU 08-025R & $-36.31 * * \pm 0.91$ & $52.82 * * \pm 0.87$ & $23.17^{*} \pm 2.85$ & $-34.31 * * \pm 2.22$ & $4.97 \pm 1.05$ \\
\hline RG-BU 08-034R & $-7.50 \pm 0.91$ & $-25.95^{*} \pm 0.87$ & $63.18^{* *} \pm 2.85$ & $16.23 \pm 2.22$ & $12.05 \pm 1.05$ \\
\hline RG-BU 08-038R & $-1.68 \pm 0.91$ & $2.86 \pm 0.87$ & $-15.59 \pm 2.85$ & $56.25^{* *} \pm 2.22$ & $29.21 * \pm 1.05$ \\
\hline RG-BU 08-046R & $112.30 * * \pm 0.91$ & $8.68 \pm 0.87$ & $13.23 \pm 2.85$ & $-22.52 \pm 2.22$ & $-0.95 \pm 0.65$ \\
\hline RG-BU 08-057R & $-5.64 \pm 0.91$ & $122.66^{* *} \pm 0.87$ & $19.04 \pm 2.85$ & $6.29 \pm 2.22$ & $-60.60 * * \pm 1.05$ \\
\hline RG-BU 08-063R & $-6.57 \pm 0.91$ & $3.79 \pm 0.87$ & $133.02 * * \pm 2.85$ & $12.11 \pm 2.22$ & $-1.96 \pm 1.05$ \\
\hline RG-BU 08-097R & $42.46^{* *} \pm 0.91$ & $12.81 \pm 0.87$ & $-27.38^{*} \pm 2.85$ & $-66.24 * * \pm 2.22$ & $15.08 \pm 1.05$ \\
\hline RG-BU 08-105R & $-15.75 \pm 0.91$ & $4.72 \pm 0.87$ & $-70.09 * * \pm 2.85$ & $41.26 * * \pm 2.22$ & $-34.31 * * \pm 1.05$ \\
\hline Minimum & $-80.03 * *$ & & & & \\
\hline Maximum & $133.02 * *$ & & & & \\
\hline SD & 24.90 & & & & \\
\hline t (5\%)/SE (gi) & 2.77 & & & & \\
\hline $\mathbf{t}(1 \%) / \mathrm{SE}(\mathrm{gi}-\mathrm{gj})$ & 24.904 & & & & \\
\hline
\end{tabular}

$* \mathrm{p}=0.05, * * \mathrm{p}=0.01$ and $^{\mathrm{ns}}=$ Insignificant

\section{Estimation of standard heterosis for yield ( $t / h a)$}

In order to formulate efficient breeding programs, for improvement of yield, it is essential to characterize the nature and mode of gene action that determines the yield and its components (Verma, 2005). It is important to know the degree and direction of hybrid vigor for its commercial exploitation (Venkatesan et al., 2007). Heterosis in yield varied from -90.83 to $189.40 \%$. Highly significant heterosis were found from the crosses of IR58025A with 6 R-lines i.e., RG-BU 08-006R (42.46**), RG-BU 08-013R (61.74**), RG-BU 08-018R (24.86*), RG-BU 08-046R (28.54**), RG-BU 08-063R (30.55**), RGBU 08-105R $(36.62 * *)$. Three crosses of IR62820A were shown exhibiting significant positive standard yield heterosis with RG-BU 08-007R (70.69**), RG-BU 08-063R (31.28**), RG-BU 08-097R (52.43**).

Seven crosses of GAN 46A showed significant positive standard yield heterosis with RG-BU 08-001R (22.45*), RG-BU 08-005R (50.04**), RG-BU 08-013R (21.82*), RG-BU 08-025 R (47.46**), RG-BU 08-038R (57.02**) and RG-BU 08-057R $\left(62.51^{* *}\right)$. Three crosses of IR $68888 \mathrm{~A}$ showed significant positive standard yield heterosis with RG-BU 08-007R (33.14**), RG-BU 08-018R (67.31**) and RG-BU 08097R $\left(22.45^{*}\right)$. And it was observed that five crosses of BRRI 1A showed significant positive standard yield heterosis with R-lines RG-BU 08-001R (41.25**), RG-BU 08005R (31.87**), RG-BU 08-016R (74.31**), RG-BU 08-025R (71.67**) and RG-BU 08-063R (31.92**). It is to ne noted that crosses of four R-lines (RG-BU 08-001R, RG-BU 08-025R, RG-BU 08-038R and RG-BU 08-097R) showed significant positive standard yield heterosis with all five CMS lines. These crosses showed marked variations in the expression of standard heterosis for yield and yield components. 
Table 7. Estimation of Standard heterosis over BRRI dhan28 for grain yield (ton/ha) in $80 \mathrm{~F}_{1}$

\begin{tabular}{|c|c|c|c|c|c|}
\hline \multirow{2}{*}{ Crosses } & \multicolumn{5}{|c|}{ Grain yield (ton/ha) } \\
\hline & IR 58025A & IR 62820A & GAN 46A & IR 68888A & BRRI 1A \\
\hline RG-BU 08-001R & $42.25^{* *}+5.21$ & $37.08 * *+5.20$ & $38.34 * *+5.21$ & $47.26^{* *+8}+8.21$ & $31.20 * *+7.21$ \\
\hline RG-BU 08-002R & $-10.37 *+2.24$ & $-9.10+3.21$ & $-5.08+3.21$ & $-11.91+3.22$ & $-11.46^{*}+1.23$ \\
\hline RG-BU 08-005R & $-15.39 * *+3.25$ & $1.40+3.27$ & $37.41 * *+7.22$ & $-4.94+3.24$ & $22.99 * *+6.25$ \\
\hline RG-BU 08-006R & $13.94 * *+3.27$ & $-14.26 * *+3.24$ & $-12.18^{*}+3.24$ & $-9.80+3.24$ & $-0.24+0.16$ \\
\hline RG-BU 08-007R & $-11.54 *+3.28$ & $54.07 * *+9.21$ & $4.60+0.29$ & $26.72 * *+1.25$ & $0.67+0.29$ \\
\hline RG-BU 08-013R & $47.89 * *+8.29$ & $-0.99+0.24$ & $15.32 * *+3.23$ & $-1.01+3.241$ & $-15.56^{*}+3.20$ \\
\hline RG-BU 08-016R & $-1.09+0.26$ & $9.55+3.21$ & $12.15^{*}+3.24$ & $-5.15+3.28$ & $55.64 * *+8.22$ \\
\hline RG-BU 08-018R & $25.67 * *+3.27$ & $15.45^{* *}+3.23$ & $8.11+3.27$ & $51.64 * *+9.22$ & $-2.86+0.20$ \\
\hline RG-BU 08-025R & $42.23^{* *}+7.22$ & $47.55^{* *}+8.25$ & $54.60 * *+3.28$ & $55.39 * *+8.20$ & $59.33 * *+9.20$ \\
\hline RG-BU 08-034R & $-4.15+0.20$ & $-4.69+3.27$ & $6.69+3.24$ & $-2.55+3.29$ & $-6.44+3.23$ \\
\hline RG-BU 08-038R & $50.59 * *+9.20$ & $36.34 * *+3.29$ & $45.33^{* *}+3.28$ & $49.41 * *+8.23$ & $55.30 * *+8.27$ \\
\hline RG-BU 08-046R & $38.60 * *+8.21$ & $-8.21+3.24$ & $-3.01+3.20$ & $-5.17+3.20$ & $1.86+1.29$ \\
\hline RG-BU 08-057R & $-14.23 * *+3.22$ & $-10.68 *+3.20$ & $51.48 * *+3.29$ & $51.41 * *+3.22$ & $49.63 * *+8.21$ \\
\hline RG-BU 08-063R & $34.89 * *+3.21$ & $34.84 * *+3.22$ & $-6.25+3.24$ & $-0.66+3.20$ & $34.04 * *+5.23$ \\
\hline RG-BU 08-097R & $92.96 * *+12.21$ & $54.58 * *+8.27$ & $51.60 * *+3.22$ & $54.34 * *+9.21$ & $35.57 * *+3.20$ \\
\hline RG-BU 08-105R & $40.45^{* *}+4.29$ & $6.93+3.20$ & $9.48+3.25$ & $4.86+3.23$ & $13.12+1.21$ \\
\hline Minimum & $-43.39 * *$ & & & & \\
\hline Maximum & $64.96 * *$ & & & & \\
\hline SD & 26.085 & & & & \\
\hline t $(5 \%) / \mathrm{SE}(\mathrm{gi})$ & 10.32 & & & & \\
\hline $\mathbf{t}(1 \%) / \mathrm{SE}(\mathrm{gi}-\mathrm{gj})$ & 13.09 & & & & \\
\hline
\end{tabular}

$* \mathrm{p}=0.05, * * \mathrm{p}=0.01$ and $^{\mathrm{ns}}=$ Insignificant

From this study it is observed that highly positive and significant cross combinations, could be exploited beneficially in future rice breeding program by adopting appropriate breeding strategy in order to evolve high yielding hybrid varieties. Occurrence of both additive and non-additive gene effects with preponderance of non-additive gene action for yield and important yield components in rice were reported by Singh 2005, Soni et al. 2005 and Tangwen et al. 2004. An importance of both additive and non-additive gene effects in governing yield and most of the yield attributes with predominance of nonadditive gene action for most of the yield attributes. In this situation, both non-additive and additive components were important for the expression of characters. At the same time population improvement program may allow to accumulate the fixable gene effects as well as to maintain considerable variability and heterozygosity for exploiting nonfixable gene effects. The higher grain yield might be due to higher amount of effective tillers/plant, higher number of filled grains/panicle and maximum out crossing rate.

\section{REFERENCES}

Ali, M. 2007. Development of parental lines for hybrid rice. Ph. D. Dissertation, Bangabandhu Sheikh Muzibur Rahman Agricultural University, Gazipur-1706, Bangladesh. 219 p.

Banumathy, S. and K. Thiyagarajan. 2005. Heterosis of rice hybrids for yield and its components. Crop Res. Hisar. 25(2): 287-293.

Bhave, S. G., B. L. Dhonukshe and V. W. Bendale. 2002. Heterosis in hybrid rice. J. Soils and crops, 12 (2): 183-186.

Chahal, G. S. and S. S. Gosal. 2008. Principles and procedures of Plant Breeding, Biotechnological and Conventional Approaches. Narosa Pub. House, New Delhi,India. Pp.247-268.

Cheita, S., C. R. A. Kumar and L. Subha. 2006. Studying heterosis for grain yield and its components in $F_{1}$ (hybrid) rice. India. Res. on Crops. 7(2): 437-439. 
Hien, N. L., W. A. Sarhadi, Y. Oikawa and Y. Hirata. 2007. Genetic diversity of morphological responses and the relationships among Asian rice (Oryza sativa L.) cultivars. Tropics. 16(4): 343-355.

Roy, A. K. 2006. Development of aromatic cytosource for hybrid rice production. Ph. D. Dissertation, Bangabandhu Sheikh Muzibur Rahman Agricultural University, Gazipur-1706, Bangladesh. 166

Singh, R. K. and S. Om. 2005. Genetic variation for yield and quality characters in mutants of rice. Annals-of-Agricultural-Research; 26(3): 406-410.

Soni, D. K., K. Arvind and S. Lakeswar. 2005. Study of heterosis by utilizing cytoplasmic-genetic male sterility system in rice (Oryza sativa L.). Plant Archives. 5(2): 617-621.

Sun, N., K. Rastogi and D. K. Soni. 2006. Genetic diversity for grain quality parameters in traditional rice (Oryza sativa L.) from Madhya Pradesh, India. Tropical Agril. Res. Extension 1(2): 103-106.

Tangwen, R., C. M. George and N. K. Dongre. 2004. Heterosis breeding in rice through line x tester design. Plant Archives. 8(2): 625-629.

Venkatesan, N .D., D. M. Maurya, G. P. Verma and S. R. Vishwakarma. 2007. Heterosis for yield components in rice hybrids (Oryza sativa). I. J. Agril. Sci. 99(7): 11201122.

Verma, O. P. and H. K. Srivastava. 2005. Heterosis and segregation distortion for grain quality traits using diverse genotypes in rice (Oryza sativa L.). Journal of Sustainable Agriculture. 26(3): 15-30. 